\title{
A New Multi-Objective Optimization in Solving Graph Coloring and Wireless Networks Channels Allocation Problems
}

\author{
Raja Marappan \\ School of Computing, SASTRA Deemed University, Thanjavur-613401 \\ Email: raja_csmath@cse.sastra.edu
} ABSTRACT-

\begin{abstract}
Graph coloring problem, a combinatorial optimization problem is being widely applied in solving the channels allocation in wireless networks. This paper exhibits a new evolutionary genetic multi-objective strategy that uses the combined single and multi-parent conflict-gene crossover and combined single and multi-parent conflict-gene mutation operators with clique partitioning to solve the graph coloring and channel allocation problems. The proposed operators minimize problem search space by reducing the expected number of genetic generations. A general fitness function is defined on finding the total conflicting edges in the graph for the initial and particularly the successive generations of individuals in the population. The outcomes of this proposed method are better than the well-known methods and are compared with some of the benchmark graph coloring and channel allocation problems. The devised method of clique partitioning with genetic operators also enhances the successful runs.
\end{abstract}

Keywords - approximation methods, channel allocation, chromatic number, genetic algorithm, graph coloring, wireless networks

\section{INTRODUCTION}

F or a simple graph $\mathrm{G}$ consists of $\mathrm{m}$ edges \& $\mathrm{n}$ vertices, the edge $\&$ vertex set are represented as $E(G):\left\{e_{1}, e_{2}, \ldots\right.$, $\left.\mathrm{e}_{\mathrm{m}}\right\}$ and $\mathrm{V}(\mathrm{G}):\left\{\mathrm{v}_{1}, \mathrm{v}_{2}, \ldots, \mathrm{v}_{\mathrm{n}}\right\}$ respectively. Also, every $\mathrm{e}_{\mathrm{i}} \epsilon$ $\mathrm{E}(\mathrm{G})$ defines a unique end vertex pair $\left(\mathrm{v}_{\mathrm{j}}, \mathrm{v}_{\mathrm{k}}\right) \in \mathrm{E}(\mathrm{G}), 1 \leq \mathrm{i}$ $\leq \mathrm{m}, 1 \leq \mathrm{j}, \mathrm{k} \leq \mathrm{n}$. The adjacency matrix of $\mathrm{G}$ is represented in $A(G)$ which is a symmetric matrix of order $\mathrm{n} \times \mathrm{n} . \mathrm{A}(\mathrm{j}, \mathrm{k})=1$ if there exists an edge joining $\mathrm{v}_{\mathrm{k}}$ and $\mathrm{v}_{\mathrm{j}}$. Otherwise, $\mathrm{A}(\mathrm{j}, \mathrm{k})$ is set to zero. Graph coloring problem (GCP) finds the least number of colors, $\chi(G)$, which are used to assign its $\mathrm{V}(\mathrm{G})$ without assigning the same colors to the adjacent vertices [1]. Recently wireless network are emerging as an important areas in which different soft computing models are required in solving the real-world problems [16]. There are many genetic methods available to find $\chi(G)$ which requires the search space of $n$ ! [2-3]. The wireless networks capacity is increased when assigning the proper $\mathrm{m}$ channels or edges to $\mathrm{n}$ cells, called Channel Assignment Problem (CAP) which is defined with the constraints: co-channel, adjacent channel, and cosite constraints (CCC, ACC \& $\mathrm{CSC})$. If $\mathrm{G}=(\mathrm{V}, \mathrm{E})$ is an undirected graph then its clique, $\mathrm{C}$, is a subgraph that consists of the vertex set $\mathrm{V}^{\prime}$, such that there is an edge between every vertex pair in $\mathrm{V}^{\prime}$. C is the complete subgraph of $\mathrm{G}$. The split of $\mathrm{G}$ into a minimum number of cliques such that each vertex belongs to exactly a single clique is called clique partition. A maximum clique is a clique that consists of the maximum number of vertices. Few other methods to find $\chi(G)$ are Particle Swarm Optimization (PSO), Ant Colony Optimization (ACO), and Cuckoo search [3]. Different methods are discussed to solve graph coloring and channel allocation problems [9$15]$.

This paper presents a new genetic method in solving GCP and CAP with a clique partition strategy. Combined Single and Multi-Parent Conflict Gene crossover (CSMPCGX), Combined Single and Multi-Parent Conflict Gene Mutation (CSMPCGM) operators are newly devised in this paper. These operators are targeted to minimize the expected genetic generations and also reduce the exploration of search space. Section II formulates the mathematical model of CAP. The evolutionary method with partitions of $\mathrm{V}(\mathrm{G})$ into different cliques in assigning proper channels is presented in Section III. Section IV analyses the theoretical results and global convergence of the devised operators. The well-known problems have experimented and their outcomes are compared with some of the existing methods and are presented in Section $\mathrm{V}$. Section VI concludes the research.

\section{FORMULATING A MATHEMATICAL MODEL OF CAP}

For the wireless network with $\mathrm{m}$ channels and $\mathrm{n}$ cells, channel assignment is an optimization problem that is defined with respect to some interference constraints. The channels assignment is defined in the vector $F$ which represents a symmetric matrix of size $\mathrm{n} \times \mathrm{m}$. For each $\mathrm{j}$ and $\mathrm{k}(1 \leq \mathrm{k} \leq \mathrm{m}$ and $1 \leq \mathrm{j} \leq \mathrm{n}), \mathrm{F}(\mathrm{j}, \mathrm{k})=1$ if cell $\mathrm{j}$ is assigned the kth channel; otherwise $F(j, k)=0$.

The demand constraint defines the maximum number of channels to be assigned to each cell, that is, the total 
assigned channels for each cell $\mathrm{i}$ should not exceed its demand value $d_{i}$. That is, $\mathrm{F}_{i 1}+\mathrm{F}_{i 2}+\mathrm{F}_{i 3}+\ldots+\mathrm{F}_{i m}-d_{i}=0$.

For any two channels $\mathrm{q}$ and $\mathrm{r}$, if $c_{i i}>|\mathrm{q}-\mathrm{r}|$, then channel $\mathrm{q}$ should not be assigned to $i^{\text {th }}$ cell. Also For any two channels $\mathrm{q}$ and $\mathrm{r}$, if channel $\mathrm{q}$ is assigned to $\mathrm{j}^{\text {th }}$ cell and if $c_{i j}>0$ and $i \neq j$ with $c_{i i}>|\mathrm{q}-\mathrm{r}|$ then $\mathrm{i}^{\text {th }}$ cell violates the assignment of the $\mathrm{q}^{\text {th }}$ channel.

The objective function of CAP is finding $\mathrm{F}$ while minimizing the overall cost function, $\mathrm{C}(\mathrm{F})$ while fulfilling the constraints of the problem.

\section{THEPROPOSEDGENETICALGORITHM}

This section presents an evolutionary method with clique partitioning in solving graph coloring and channel allocation problems and its flowchart is presented in Figure 1. Cliques are obtained and are assigned the proper channels in minimizing the network interference. The strategy splits $G$ into several maximum cliques. For the interfering cliques, different channels are assigned to them. The overall skeleton to find the solution to CAP is given below:

i. Apply partition of $\mathrm{V}(\mathrm{G})$ to obtain the different cliques, say $c_{1}, c_{2}, c_{3} \ldots c_{q}$.

ii. Apply the following for each of the cliques $\mathrm{i}=1$ to $\mathrm{q}$ :

a. Find the feasible solution for vertex partitions and cliques.

b. Arbitrarily check for the interference of any two cliques and assign the valid channel to the cells based on interference constraints.

Clique partition constructs a super graph G': (S, E') which is obtained from $\mathrm{G}$ : $(\mathrm{V}, \mathrm{E})$. Every vertex $\mathrm{s}_{\mathrm{i}}$ in $\mathrm{S}$ is a super vertex that consists of a set of finite vertices $v_{i}$ in $V$. A vertex $s_{i}$ in $S$ is a common neighbor of $s_{j}$ and $s_{k}$ in $S$ if there exist edges $(i, j)$ and $(i, k)$ in $E$ '. The following procedure finds the maximum clique of $\mathrm{G}$ :

a. Put each $v_{i}$ in $V(G)$ in a separate vertex $s_{i}$ in $S$ of G'.

b. Identify $s_{i 1}$ and $s_{i 2}$ in $S$ such that $\left(s_{i 1}, s_{i 2}\right)$ in $E^{\prime}(G)$ with the greatest number of common adjacent vertices.

c. Join the identified vertices into a single vertex $\mathrm{s}_{\mathrm{i} 1 \mathrm{i} 2}$ which have $\mathrm{s}_{\mathrm{i} 1}$ and $\mathrm{s}_{\mathrm{i} 2}$ in all.

d. Determine the general set which consists of the entire general neighbors of $\mathrm{s}_{\mathrm{i} 1}$ and $\mathrm{s}_{\mathrm{i} 2}$.

e. Delete all edges which are emerging from $\mathrm{s}_{\mathrm{i} 1}$ or $\mathrm{s}_{\mathrm{i} 2}$ in $\mathrm{G}^{\prime}$.

f. Include the new $e_{i}$ 's from $s_{i 12}$ to every super vertex in the general set.

g. Iteratively execute the procedure defined in (a) to (f) until $G$ has no edges.

h. Vertex set $\mathrm{s}_{\mathrm{i}}$ in $\mathrm{S}$ defines the clique.

Flowchart to solve GCP \& CAP using the proposed method is shown in Figure 1. The new genetic operators are represented below.

\section{CSMPCGXoperation:}

Apply steps (a) to (e) of SPCGX crossover [11].
Apply crossover at single, multiple points and find the better offspring.

Repeat the combined single and multipoint crossover for $t$ times.

Obtain the offspring: $i^{\prime}=\left(i_{1}, i_{2}, i_{3}, \ldots i_{n}\right) \& j^{\prime}=\left(j_{1}\right.$, $\left.\mathrm{j}_{2}, \mathrm{j}_{3^{\prime}}, \ldots \mathrm{j}_{\mathrm{n}^{\prime}}\right)$.

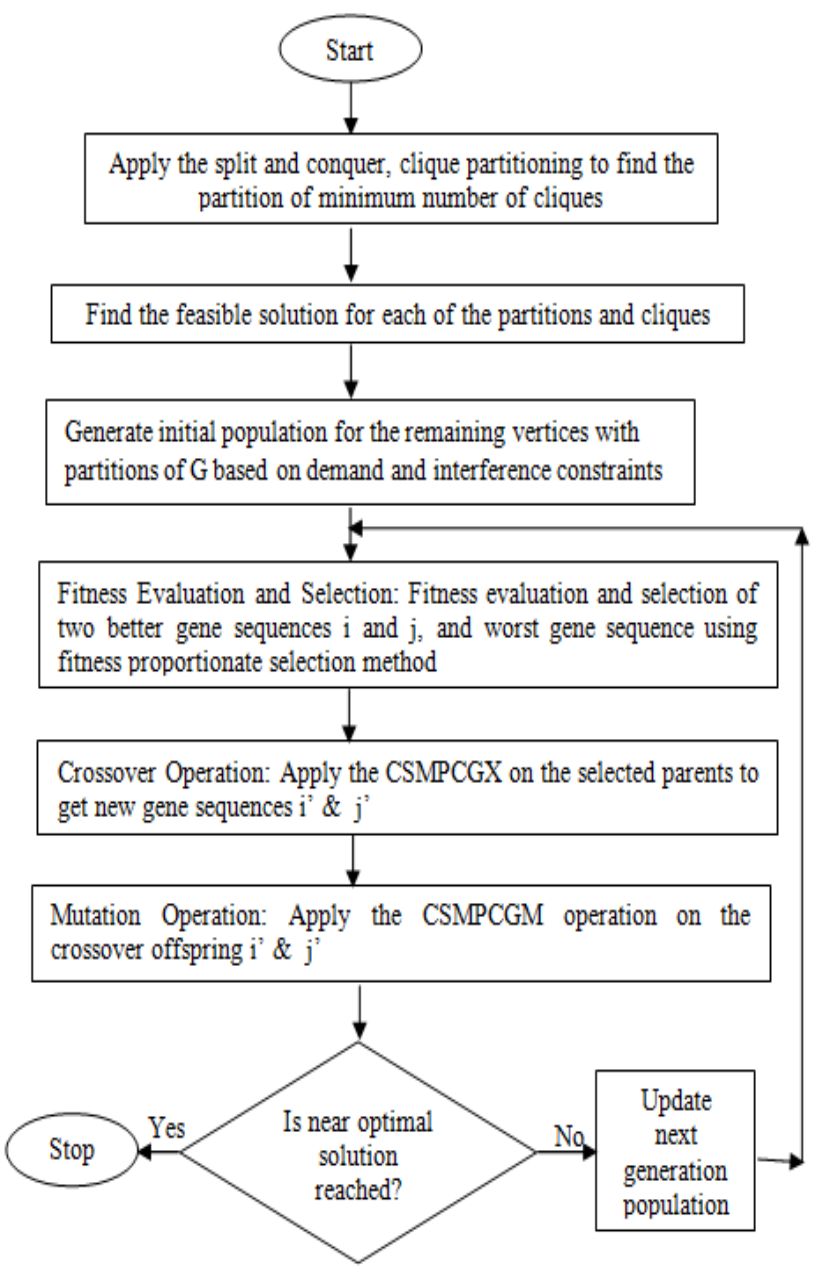

Fig. 1: Solving GCP \& CAP using the proposed method - Flowchart

\section{CSMPCGMoperation:}

Apply the steps (a) to (d) of the mutation operator [11]. Apply mutation at single, multiple points and find the better offspring.

Repeat the combined single and multipoint crossover for $\mathrm{t}$ times.

Generate better offspring: i" and $\mathrm{j}$.

\section{ANALYSIS \\ CONVERGENCE}

AND

GLOBAL

This section focus on the analysis and global convergence of the proposed genetic operators. The devised operators achieve quick stochastic convergence in finding the better near optimal solution. The stochastic convergence of the proposed operators are analyzed as follows:

The new genetic operators with clique partition strategy monotonically reduce the fitness values of gene sequences 
$i$ and $j, f_{g}(i) \& f_{g}(j)$ and the following analysis is observed during the generations:

Case (a): Either $\mathrm{f}_{\mathrm{g}}(\mathrm{i})$ or $\mathrm{f}_{\mathrm{g}}(\mathrm{j})$ decreases monotonically and converges. In this case,

$$
f_{0}(i) \geq f_{1}(i) \geq f_{2}(i) \geq \ldots \geq f_{q}(i) \geq f_{q+1}(i)=0 \text { or } f_{0}(j) \geq
$$$$
f_{1}(j) \geq f_{2}(j) \geq \ldots \geq f_{q}(j) \geq f_{q+1}(j)=0 \text {. }
$$

Case (b): At the initial stage, if $f_{g}(i)$ or $f_{g}(j)$ satisfies $f_{0}(i) \geq$ $f_{1}(i) \geq f_{2}(i) \geq \ldots \geq f_{q}(i)$ and $f_{0}(j) \geq f_{1}(j) \geq f_{2}(j) \geq \ldots \geq f_{q}(j)$, then after finite and smaller iterations, the following holds to converge.

$$
\mathrm{f}_{\mathrm{q}}(\mathrm{i})<\mathrm{f}_{\mathrm{q}+1}(\mathrm{i})<\ldots<\mathrm{f}_{\mathrm{t}}(\mathrm{i}) \text { and } \mathrm{f}_{\mathrm{q}}(\mathrm{j})<\mathrm{f}_{\mathrm{q}+1}(\mathrm{j})<\ldots<\mathrm{f}_{\mathrm{t}}(\mathrm{j})
$$

The global convergence of the devised method to achieve better near optimal solution while reducing the search space is proved in the following theorem.

\section{Theorem 1: The proposed genetic algorithm with CSMPCGX and CSMPCGM always converges stochastically.}

Proof: Let $\mathrm{S}$ represents the finite search space. The following conditions should be fulfilled for stochastic convergence [2]. For arbitrarily chosen individuals $i, j \in S$, individuals should be reachable and the population $\mathrm{P}_{g}$ satisfy the monotone property.

$\mathrm{p}_{\mathrm{c}}$ and $\mathrm{p}_{\mathrm{m}}$ define the CSMPCGX \& CSMPCFM probabilities for applying crossover and mutation respectively.

Then $\mathrm{p}\left(\mathrm{i}{ }^{\prime}\right) \geq \mathrm{p}_{\mathrm{c}} \mathrm{p}\left\{\mathrm{i}^{\prime}=\operatorname{crossover}(\mathrm{i})\right\} \mathrm{p}_{\mathrm{m}} \mathrm{p}\left\{\mathrm{i}{ }^{\prime}=\right.$ mutation (i')\}

CSMPCGX operation implies $\mathrm{p}\left\{\mathrm{i}^{\prime}=\operatorname{crossover}(\mathrm{i})\right\}=1 / \mathrm{c}^{\mathrm{k}}$ $>0$ and

CSMPCGM results in $\mathrm{p}\left\{\mathrm{i}^{\prime \prime}=\operatorname{mutation}\left(\mathrm{i}^{\prime}\right)\right\}=1 / \mathrm{f}(\mathrm{G})^{\mathrm{l}}>0$. Thus $p\left(i^{\prime} '\right) \geq\left(p_{c} p_{m}\right) /\left(c^{k} f(G)^{1}\right)$ is in $[0,1]$.

The elitism operation replaces the worst individual with a better one and $\mathrm{P}_{\mathrm{g}}$ gets updated for the monotone property.

\section{RESULTS OF BENCH MARK INSTANCES}

The maximum number of cells considered is 25 and the maximum number of channels considered for the simulation of the proposed method is 91 . The devised operators are tested in solving some standard benchmark graphs and CAPs using Intel Core i5-2450M $2.5 \mathrm{GHz}$ system using Java. The graph parameters $\mathrm{n}$ and $\mathrm{m}$ are evaluating the output performance measurements. The outcomes are analyzed with the following inferences.

1. Stochastic convergence is reached even to the highdensity graphs, such as miles1500.col.

2. Expected generations are reduced compared to other genetic methods and the obtained values are shown in figures, Fig. 2 to Fig. 4.

3. The solution is obtained with minimal complexity for the queen, register allocation, Miles graphs.

4. When the number of cells increase, the frequency of convergence will be affected.

5. The frequency of convergence depends on the number of channels. When the channels count decreases, near optimal solution with less frequency of convergence is attained.

When comparing the results of this method with some of the existing methods [9-15], this new method offsets the problems such as improving the near-optimal solution, minimize the problem search space, and achieve fast stochastic convergence with smaller population size $\mathrm{N}$. The major inferences to target these justifications are:

1. The solution is obtained for different benchmark random graphs.

2. Stochastic convergence is achieved for small $\mathrm{N}$ compared to other methods [9-21].

3. The solution is obtained for queen, Mycielski graphs compared to the near-optimal performance of existing methods.

4. Provides better solution than DSATUR, FINOCCHI, and FROGSIM methods.

Table 1 represents the different CAP instances and the expected generations obtained are given in Figure 5. The convergence of near-optimal solutions for these problems is shown in Table II and these outcomes are matched with some existing methods [4-7] and are indicated in Table III. This new method significantly reduces the complexity in getting the solution with a minimum fitness threshold and higher frequency of convergence (FOC).

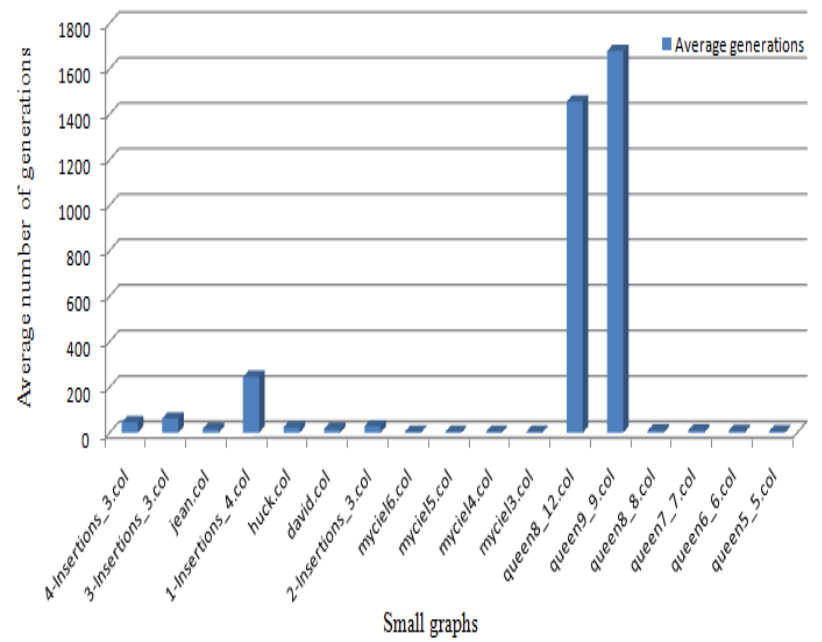

Fig. 2 Expected generations obtained for small graphs

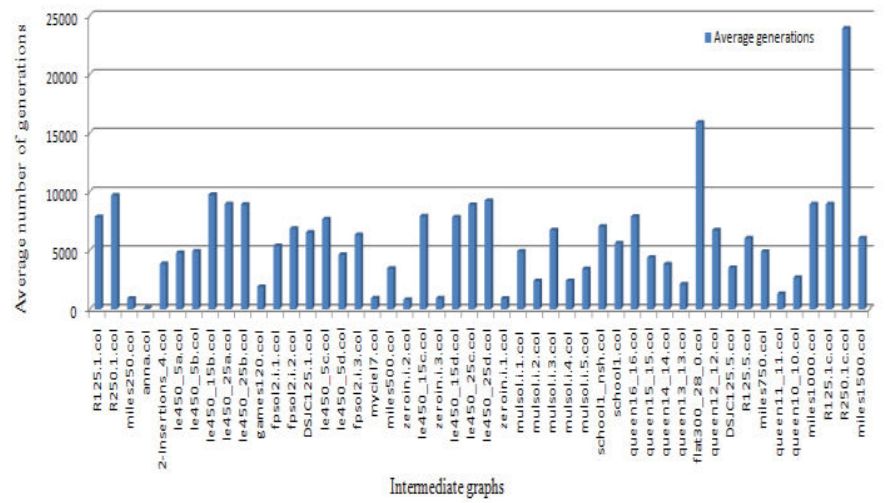

Fig 3. Expected generations obtained for intermediate graphs 


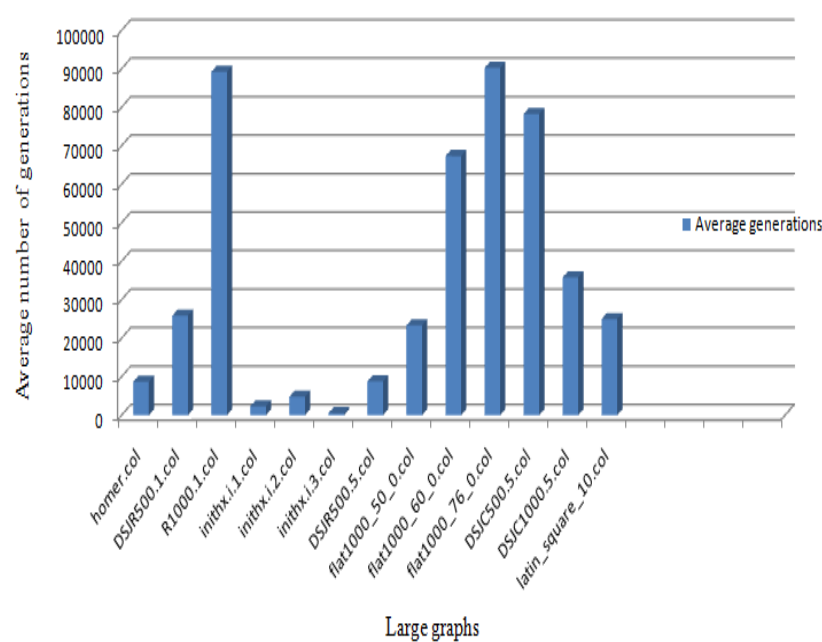

Fig. 4 Expected generations obtained for large graphs

Table I C(F) obtained for various benchmark instances

\begin{tabular}{|l|l|l|l|}
\hline Problem & Number of Cells & Number of Channels & C(F) \\
\hline EX1 & 4 & 11 & 0 \\
\hline Ex2 & 5 & 17 & 0 \\
\hline HEX1 & 21 & 37 & 0 \\
\hline HEX2 & 21 & 91 & 0 \\
\hline HEX3 & 21 & 21 & 0 \\
\hline HEX4 & 21 & 56 & 0 \\
\hline KUNZ1 & 10 & 30 & 0 \\
\hline KUNZ2 & 15 & 44 & 0 \\
\hline KUNZ3 & 20 & 60 & 0 \\
\hline KUNZ4 & 25 & 73 & 0 \\
\hline & & &
\end{tabular}

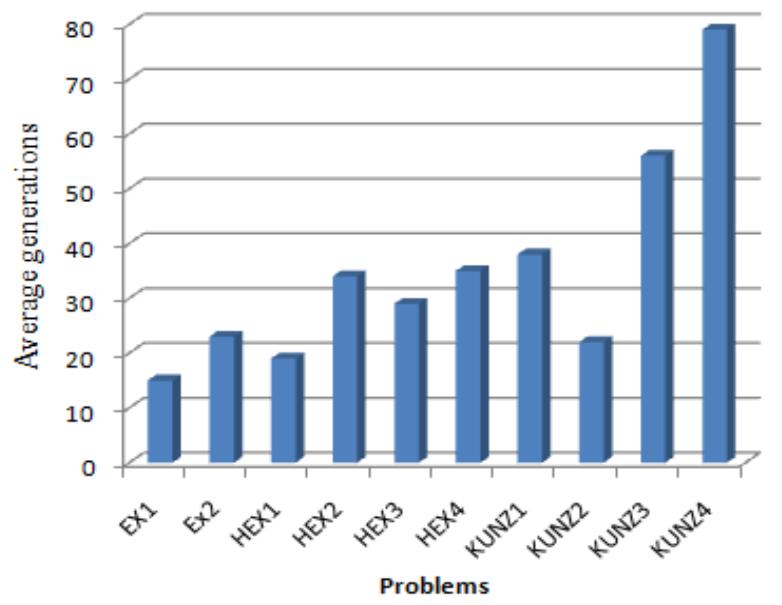

Fig. 5 Expected generations obtained for CAP instances
Table II Convergence of $\mathrm{F}$ for benchmark instances

\begin{tabular}{|c|c|c|c|c|c|}
\hline Problem & Cells n & $\begin{array}{c}\text { Channels } \\
\mathrm{m}\end{array}$ & $\begin{array}{c}\text { Simulation } \\
\text { Run Length }\end{array}$ & $\begin{array}{c}\text { Fitness } \\
\text { Threshold }\end{array}$ & FOC \\
\hline 1 & 4 & 11 & 100 & 0 & $100 \%$ \\
\hline 2 & 25 & 73 & 20000 & 0 & $100 \%$ \\
\hline 3 & 21 & 385 & 20000 & 0 & $100 \%$ \\
\hline 4 & 500 & 1250 & 40000 & 0.83 & $100 \%$ \\
\hline 5 & 1000 & 1500 & 45000 & 0.89 & $100 \%$ \\
\hline 6 & 2000 & 1750 & 50000 & 3.45 & $99.5 \%$ \\
\hline 7 & 5000 & 2000 & 50000 & 2.48 & $98.6 \%$ \\
\hline 8 & 10000 & 2500 & 50000 & 6.46 & $97.9 \%$ \\
\hline 9 & 20000 & 5000 & 50000 & 7.92 & $97.5 \%$ \\
\hline 10 & 50000 & 7500 & 50000 & 9.95 & $98.5 \%$ \\
\hline
\end{tabular}

Table III Comparison with Existing Methods

\begin{tabular}{|c|c|c|c|c|}
\hline \multirow{2}{*}{ Problem } & $\begin{array}{c}\text { Existing } \\
\text { Methods }\end{array}$ & \multicolumn{3}{|c|}{ Proposed Method } \\
\cline { 2 - 5 } & FOC & FOC & $\begin{array}{c}\text { No. of } \\
\text { Trials }\end{array}$ & Time(sec) \\
\hline 1 & $100 \%$ & $100 \%$ & 1 & 0 \\
\hline 2 & $92 \%$ & $100 \%$ & 6 & 36 \\
\hline 3 & $80 \%$ & $100 \%$ & 1 & 0 \\
\hline 4 & - & $100 \%$ & 23 & 762 \\
\hline 5 & - & $100 \%$ & 545 & 864 \\
\hline 6 & - & $99.5 \%$ & 453 & 342 \\
\hline 7 & - & $98.6 \%$ & 564 & 654 \\
\hline 8 & - & $97.9 \%$ & 233 & 544 \\
\hline 9 & - & $97.5 \%$ & 348 & 889 \\
\hline 10 & - & $98.5 \%$ & 655 & 903 \\
\hline
\end{tabular}

\section{CONCLUSION}

A new genetic method is designed for solving GCP \& CAP using CSMPCGX \& CSMPCGM operators. The experiments are conducted on difficult benchmark instances and showed that this method outperforms the various existing methods with the reduction in the exploration of search space and minimizing the expected generations. This evolutionary strategy reduces the interference of CAP with minimal $\mathrm{N}$ and hence better performance is obtained compared to the well-known methods. Stochastic convergence of higher frequency of convergence is attained for the standard and random CAPs of large sizes.

\section{REFERENCES}

[1] L.Mumford: New order-based crossover for the graph coloring problem, T. P. Runarsson et al. (Eds.): PPSN LX, vol. 4193, pp. 80-88, 2006.

[2] G. Rudolph: Finite Markov chain results in evolutionary computation: A tour Horizon, Fundamenta Informaticae, vol. 35, no.2, pp. 67-89.

[3] Ling-Yuan Hsu; Shi-Jinn Horng; Pingzhi Fan; Muhammad Khurram Khan; Yuh-Rau Wang; Ray- 
Shine Run; Jui-Lin Lai; Rong-Jian Chen: MTPSO algorithm for solving planar graph coloring problem. Expert Syst. Appl., 38:5525-5531, May 2011.

[4] Shih Heng Cheng; Ching Yao Huang: Coloring-Based Inter-WBAN Scheduling for Mobile Wireless Body Area Networks, IEEE Transactions on parallel and distributed systems, vol. 24, no. 2, 2013.

[5] Elmahdi Driouch; Wessam Ajib: Downlink Scheduling and Resource Allocation for Cognitive Radio MIMO Networks, IEEE Transactions on vehicular technology, vol. 62, no. 8, 2013.

[6] Elmahdi Driouch; Wessam Ajib: Efficient Scheduling Algorithms for Multiantenna CDMA Systems, IEEE Transactions on vehicular technology, vol. 61, no. 2, 2012.

[7] Matti Peltomäki; Juha-Matti Koljonen; Olav Tirkkonen; Mikko Alava:: Algorithms for SelfOrganized Resource Allocation in Wireless Networks, IEEE transactions on vehicular technology, vol. 61, no. $1,2012$.

[8] The Graph Coloring instances, http://mat.gsia.cmu.edu/COLOR/instances.html.

[9] Soma Saha; Rajeev Kumar; Gyan Baboo: Characterization of graph properties for improved Pareto fronts using heuristics and EA for bi-objective graph coloring problem, Applied Soft Computing, ASOC-1644, 2012.

[10] Sethumadhavan, G; Marappan, R.. A Genetic Algorithm for Graph Coloring using Single Parent Conflict Gene Crossover and Mutation with Conflict Gene Removal Procedure, 2013 IEEE International Conference on Computational Intelligence and Computing Research, India, pages 350-355, 26-28 December 2013.

[11] Marappan, R.; Sethumadhavan, G. Solving Graph Coloring Problem for Large Graphs, Global Journal of Pure and Applied Mathematics, ISSN 0973-1768 Volume 11, Number 4 (2015), pp. 2487-2494.

[12] Marappan, R.; Sethumadhavan, G. Solving Fixed Channel Allocation using Hybrid Evolutionary Method. ICAET, MATEC Web of Conferences 57, 02015 (2016)

[13] Marappan, R.; Sethumadhavan, G. Solving Channel Allocation Problem using New Genetic Algorithm with Clique Partitioning Method. IEEE International Conference on Computational Intelligence and Computing Research (ICCIC 2016) (2017)

[14] Marappan, R.; Sethumadhavan, G. Solution to Graph Coloring Using Genetic and Tabu Search Procedures. Arabian Journal for Science and Engineering, DOI 10.1007/s13369-017-2686-9 (2019)

[15] Marappan, R.; Sethumadhavan, G. Complexity analysis and stochastic convergence of some wellknown evolutionary operators for solving graph coloring problem. Mathematics 2020, 8, 303. doi: https://doi.org/10.3390/math8030303.

[16] Mallikarjunaswamy N J; Latha Yadav T R; Dr. Keshava Prasanna: Message Authentication Protocol for Lifetime Proficient Hash Based Algorithm in Wireless Sensor Networks. Int. J. Advanced
Networking and Applications. Volume: 07 Issue: 06 Pages: 2963-2966 (2016) ISSN: 0975-0290

\section{AUTHOR'S BIOGRAPHY}

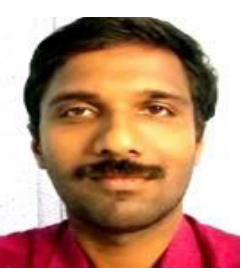

Raja Marappan is currently Senior Assistant Professor with the School of Computing, SASTRA Deemed University, Thanjavur, India with more than 21 years of teaching experience and more than 7 years of research experience. He received a Master of Technology (M.Tech.) in Computer Science \& Engineering, first-class with distinction, from SASTRA University, in January 2009. He successfully qualified for the University Grants Commission - National Eligibility Test (UGC-NET) exam in June 2012. He completed his Doctor of Philosophy (Ph.D.) in Computer Science \& Engineering from SASTRA University, in August 2018. His research focuses on the design and analysis of soft computing algorithms in solving NP-hard and NPcomplete problems. His research interests also include algorithmic computing, evolutionary computing, simulation, and modeling. He has published more than 12 papers in SCOPUS-indexed journals and 5 papers in SCIE indexed journals since 2012 and also won the best paper award at the IEEE International Conference on Computational Intelligence and Computing Research in 2013. 